\title{
Leptonic and Hadronic Modeling of Fermi-Detected Blazars
}

\section{Spectral Energy Distribution Modeling and High-Energy Polarization Predictions}

\author{
Markus Böttcher ${ }^{1,2, a}$, Anita Reimer ${ }^{3, b}$, and Haocheng Zhang ${ }^{2, c}$ \\ ${ }^{1}$ Centre for Space Research, North-West University, Potchefstroom, 2531, South Africa \\ ${ }^{2}$ Astrophysical Institute, Department of Physics and Astronomy, Ohio University, Athens, $\mathrm{OH} 45701$, USA \\ ${ }^{3}$ Institut für Theoretische Physik, Universität Innsbruck, Technikerstraße 25, A-6020, Innsbruck, Austria
}

\begin{abstract}
We describe new implementations of leptonic and hadronic models for the broadband emission from relativistic jets in AGN in a temporary steady state. The new model implementations are used to fit snap-shot spectral energy distributions of a representative set of Fermi-LAT detected blazars from the first LAT AGN catalogue. We find that the leptonic model is capable of producing acceptable fits to the SEDs of almost all blazars with reasonable parameters close to equipartition between the magnetic field and the relativistic electron population. If charge neutrality in leptonic models is provided by cold protons, our fits indicate that the kinetic energy carried by the jet should be dominated by protons. We also find satisfactory representations of the snapshot SEDs of most blazars in our sample with the hadronic model presented here. All of our hadronic model fits require powers in relativistic protons in the range $10^{47}-10^{49} \mathrm{erg} / \mathrm{s}$. As a potential way to distinguish between the leptonic and hadronic high-energy emission models considered here, we suggest diagnostics based on the predicted X-ray and $\gamma$-ray polarization, which are drastically different for the two types of models.
\end{abstract}

\section{Introduction}

The spectral energy distributions (SEDs) of blazars are characterized by non-thermal continuum spectra with a broad low-frequency component in the radio - UV or Xray frequency range (generally agreed to be due to synchrotron radiation of relativistic electrons) and a highfrequency component from X-rays to $\gamma$-rays. Blazars are sub-divided based on the location of their synchrotron peak: Low-Synchrotron-Peaked (LSP) blazars, consisting of FSRQs and Low-frequency-peaked BL Lac objects (LBLs) have $v_{\mathrm{sy}}<10^{14} \mathrm{~Hz}$; Intermediate-SynchrotronPeaked (ISP) blazars (which are exclusively BL Lac objects and hence also termed IBLs for Intermediate BL Lac Objects) have $10^{14} \mathrm{~Hz} \leq v_{\mathrm{sy}} \leq 10^{15} \mathrm{~Hz}$; HighSynchrotron-Peaked (HSP) blazars (also exclusively BL Lac objects, termed HBLs for High-peaked BL Lac objects) have $v_{s}>10^{15} \mathrm{~Hz}$.

The extreme inferred isotropic luminosities, combined with the rapid high-energy variability, provide convincing evidence that the high-energy emission from blazars originates in relativistic jets closely aligned with our line of sight [for a review of those arguments, see, e.g., 23]. The simplest (and often sufficient) assumption is that the emission is produced in an approximately spherical region, propagating along the jet with a speed $\beta_{\Gamma} c$, corresponding to a bulk Lorentz factor $\Gamma$. If the jet forms an

\footnotetext{
a e-mail: Markus.Bottcher@nwu.ac.za

be-mail: Anita.Reimer@uibk.ac.at

ce-mail: zhangh@phy.ohiou.edu
}

angle $\theta_{\text {obs }}$ with respect to our line of sight, this results in Doppler boosting characterized by the Doppler factor $D=\left(\Gamma\left[1-\beta_{\Gamma} \cos \theta_{\text {obs }}\right]\right)^{-1}$ (for a review of relativistic effects in the jets of AGN, see [6]).

While it is generally accepted that the low-frequency emission component in blazars SEDs is synchrotron emission from relativistic electrons in the jet, the origin of the high-energy emission is still controversial. In leptonic models, the radiative output throughout the electromagnetic spectrum is assumed to be dominated by leptons, while any protons that are likely present in the outflow, are not accelerated to sufficiently high energies to contribute significantly to the radiative output. The high-energy emission is then most plausibly explained by Compton scattering of low-energy photons by the same electrons producing the synchrotron emission at lower frequencies $[3,4,11,16,24]$. In hadronic models, both primary electrons and protons are accelerated to ultrarelativistic energies, with protons exceeding the threshold for $\mathrm{p} \gamma$ photopion production on the soft photon field in the emission region. While the low-frequency emission is still dominated by synchrotron emission from primary electrons, the high-energy emission is dominated by proton synchrotron emission, $\pi^{o}$ decay photons, synchrotron and Compton emission from secondary decay products of charged pions, and the output from pair cascades initiated by these highenergy emissions intrinsically absorbed by $\gamma \gamma$ pair production $[1,15,17,18]$. For a general overview of the features of both leptonic and hadronic blazar models, see [5]. 
In a recent paper [7], we have developed efficient implementations of stationary, one-zone leptonic and hadronic emission models for blazars, which allowed for a systematic, fair comparison of leptonic and hadronic model fits of a sample of Fermi-LAT detected blazars. As we will elaborate in more detail below in section 2 , one of the salient results of this comparative model fitting is that typically both leptonic and hadronic models are capable of providing satisfactory fits to simultaneous snap-shot SEDs of many blazars.

Two possibilities to distinguish leptonic from hadronic models for blazar emission have been amply discussed in the literature: (1) Only hadronic models predict the emission of neutrinos from the $\gamma$-ray emission region, as a result of charged-pion and subsequent muon decays. The detection of neutrinos from blazars would therefore uniquely prove the importance of hadronic processes in these objects. However, model predictions of the neutrino fluxes expected in hadronic blazar models are typically below the sensitivities even of IceCube or KM3NET [e.g., 18, 21]. (2) Leptonic and hadronic models may produce very different variability signatures. While very rapid (minutescale) variability observed in several blazars poses severe problems for hadronic models, apparently uncorrelated optical, X-ray and $\gamma$-ray variability is more readily explained in hadronic models [12, 25].

As an alternative to those two possibilities, we [26] have recently explored the X-ray and $\gamma$-ray polarization signatures from the leptonic and hadronic blazar models considered in [7], and found that they are drastically different. In view of initial success of measuring hard X-ray / soft $\gamma$-ray polarization of a few gamma-ray bursts with INTEGRAL [10, 13], the current development of baloonborne X-ray polarimeters [2, 19, 20], and the potential prospect of measuring $\gamma$-ray polarization at $E<200 \mathrm{MeV}$ with Fermi-LAT [9], we are suggesting X-ray and $\gamma$-ray polarization measurements as an alternative diagnostic to distinguish leptonic from hadronic high-energy emission from blazars.

In Section 2, we briefly summarize our newly developed stationary leptonic and hadronic one-zone blazar models and the results of comparative leptonic and hadronic model fitting of a number of Fermi-LAT detected blazars. Our calculations of the X-ray and $\gamma$-ray polarization expected in these models are described in Section 3 , and in Section 4, we suggest an observational strategy, based on optical, X-ray, and $\gamma$-ray polarization measurements, to distinguish between leptonic and hadronic highenergy emission from blazars.

\section{Leptonic and Hadronic Model fits to Blazar SEDs}

In [7], we have developed efficient codes for stationary one-zone leptonic and hadronic jet models for blazars. In both models, the energy distributions of the radiating particles are determined self-consistently as a balance between injection (parameterized by injection luminosities $L_{e}$ and $L_{p}$ ), radiative and adiabatic energy losses, and particle es-
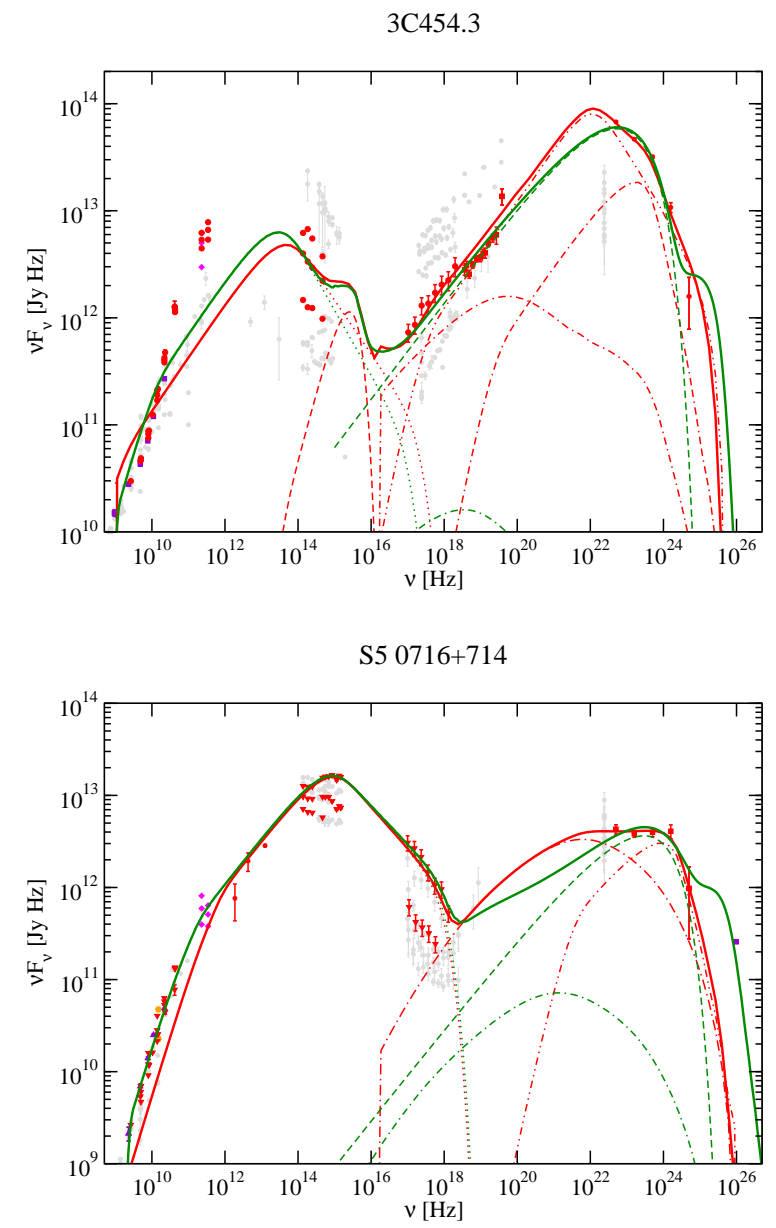

Figure 1. Leptonic (red) and hadronic (green) model fits to the SED of the FSRQ 3C454.3 (top) and the LBL S5 0716+714 (bottom)

cape from the emission region. For the hadronic model, the energy spectra of the final decay products resulting from $\mathrm{p} \gamma$ pion production are calculated using the templates of [14], and we have developed a semi-analytical approach to evaluating the radiative output from $\gamma \gamma$ absorption induced, synchrotron-supported pair cascades.

Figure 1 shows two typical examples of the resulting leptonic and hadronic fits to the SEDs of the FSRQ 3C 454.3 and the LBL S5 0716+714. In summary, both leptonic and hadronic one-zone models are generally able to provide reasonable fits to the SEDs of most FermiLAT detected blazars. Leptonic fits require characteristic kinetic powers in relativistic electrons of $L_{e}^{\text {lept }} \sim$ a few $\times 10^{44}$ ergs $\mathrm{s}^{-1}$, in many cases with magnetic fields of $B^{\text {lept }} \sim 1-$ a few $\mathrm{G}$, near equipartition with the relativistic electron population. If charge neutrality is provided by non-relativistic protons in the jet, the jets are proton dominated, with kinetic luminosities in protons of $L_{p}^{\text {lept }} \sim 5-$ $50 L_{e}^{\text {lept }}$.

The power requirements for hadronic models are substantially higher than for leptonic models, with $L_{p}^{\text {had }} \sim 10^{48}$ - a few $\times 10^{49}$ ergs s $^{-1}$, and the jets are always strongly dominated by the kinetic power in protons, in spite of characteristic magnetic fields of $B^{\text {had }} \sim 10-100 \mathrm{G}$. 
$3 \mathrm{C} 454.3$

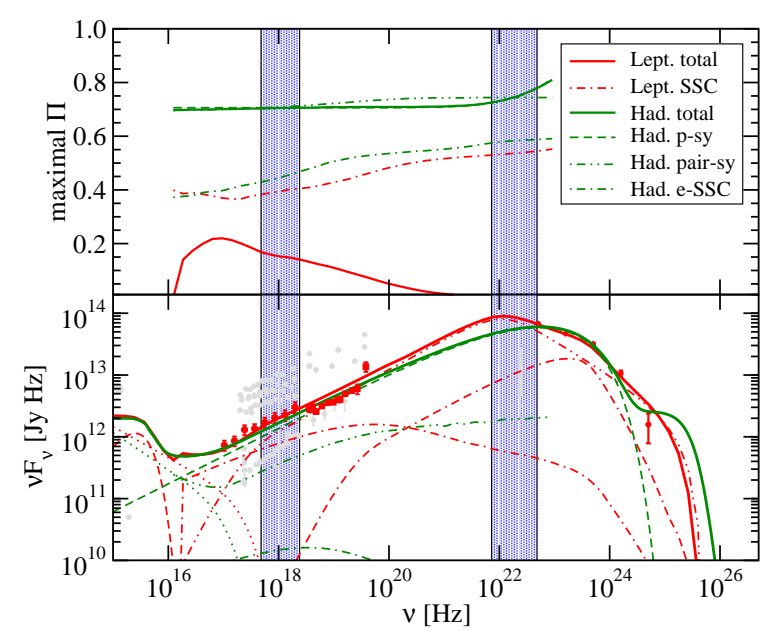

S5 $0716+714$

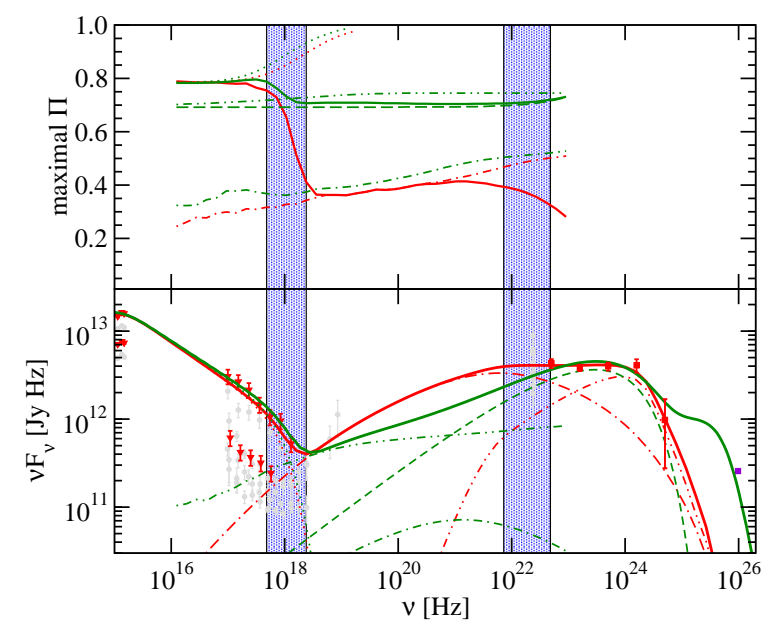

Figure 2. Lower panels: Zoom-in on the UV through $\gamma$-ray SEDs and leptonic (red) and hadronic (green) spectral fits to the FSRQ 3C454.3 (top) and the LBL S5 0716+714 (bottom). Top panels: Frequency-dependent maximum degree of polarization corresponding to the spectral fits presented in the lower panels.

\section{High-Energy Polarization Predictions}

As a potential diagnostic to distinguish between leptonic and hadronic high-energy emission in blazars, we [26] have recently investigated the polarization signatures resulting from both classes of models. Since leptonic and hadronic models agree on the interpretation of the lowfrequency (radio through UV or X-ray) emission as being due to synchrotron radiation of relativistic electrons, they make identical predictions for the radio through optical polarization from blazars. It can therefore only be in the high-energy (X-ray and $\gamma$-ray) polarization that the predictions of both models diverge.

In leptonic models, the X-ray through $\gamma$-ray emission results from electron synchrotron radiation (in X-rays for HSP blazars), synchrotron self-Compton (SSC), and external-Compton (EC) radiation. The photon-energy dependent degree of synchrotron polarization can be eval- uated with the well-known standard expressions to be found, e.g., in [22]. In the relevant energy range below $200 \mathrm{MeV}$, in which $\gamma$-ray polarization may be measurable with Fermi-LAT, Compton scattering happens safely in the Thomson regime, for which the expressions of [8] may be used to evaluate the resulting degree of polarization. As a rule of thumb, the degree of polarization of SSC radiation is about half the degree of polarization of the target synchrotron photon field. Compton scattering of unpolarized external radiation fields will yield unpolarized X-ray $-\gamma$-ray emission due to the azimuthal symmetry of the scattering geometry.

In hadronic models, the $\mathrm{X}$-ray $-\gamma$-ray emission is a superposition of synchrotron emissions from primary electrons, relativistic protons, and secondary particles from ultra-high-energy $\gamma$-ray induced pair cascades $(\gamma$ rays from $\pi^{o}$ decay are produced at too high energies to escape from the emission region). For all species, the standard expressions of [22] are used to evaluate the photonenergy dependent degree of polarization.

For both leptonic and hadronic models, we have calculated upper limits to the expected degree of polarization by assuming a perfectly ordered, homogeneous magnetic field in the emission region, oriented perpendicular to the line of sight. The degree of polarization for various blazars has been calculated based on the relativistic particle distributions required for the SED fits for those blazars using the codes of [7]. Figure 2 shows two characteristic examples LSP blazars, while Figure 3 shows the same for an ISP and an HSP blazar.

Depending on the type of blazar (LSP, ISP, HSP), the primary-electron synchrotron radiation may make a contribution to the X-ray emission, in which case both leptonic and hadronic models make essentially identical predictions for the maximum degree of polarization. Towards higher frequencies, however, hadronic models predict consistently higher degrees of polarization than leptonic ones, and the difference is most drastic at $\gamma$-ray energies. While hadronic models predict upper limits to the degrees of $\gamma$ ray polarization up to $\sim 75-80 \%$, this value does typically not exceed $\sim 35 \%$ for SSC-dominated $\gamma$-ray emission, and EC-dominated $\gamma$-ray emission is expected to be entirely unpolarized.

\section{High-Energy Polarization as Observational Diagnostic of Leptonic vs. Hadronic $\gamma$-ray emission}

The results shown in Figures 2 and 3 assume a perfectly ordered magnetic field and therefore only represent upper limits to the possible degree of polarization actually observed. In order to obtain a more realistic prediction of the $\mathrm{X}$-ray and $\gamma$-ray polarization, one needs to know the degree of order/disorder of the magnetic field. This may be determined by measuring the degree of polarization in energy ranges of the SED that can be confidently ascribed to synchrotron emission, for which the maximum degree of polarization can be readily calculated. Comparing the 


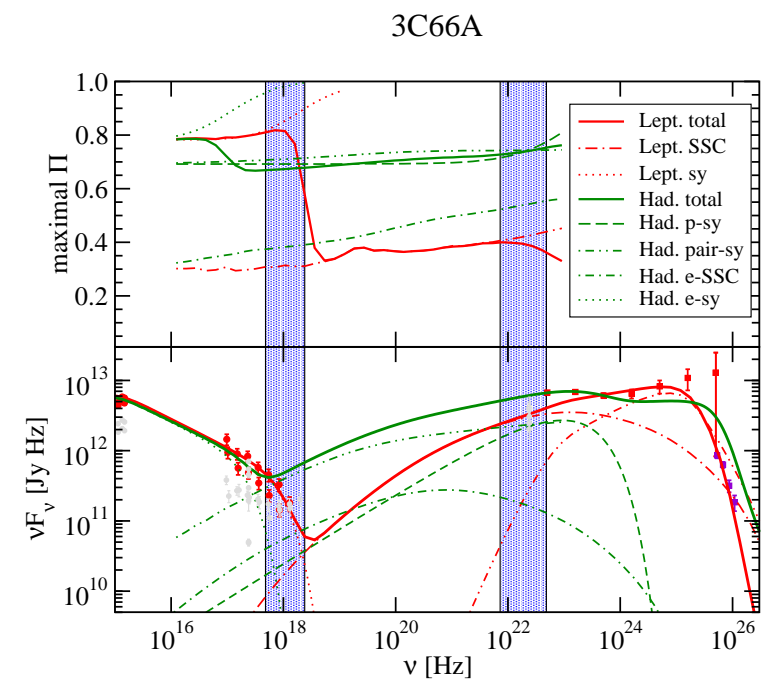

RBS 0413

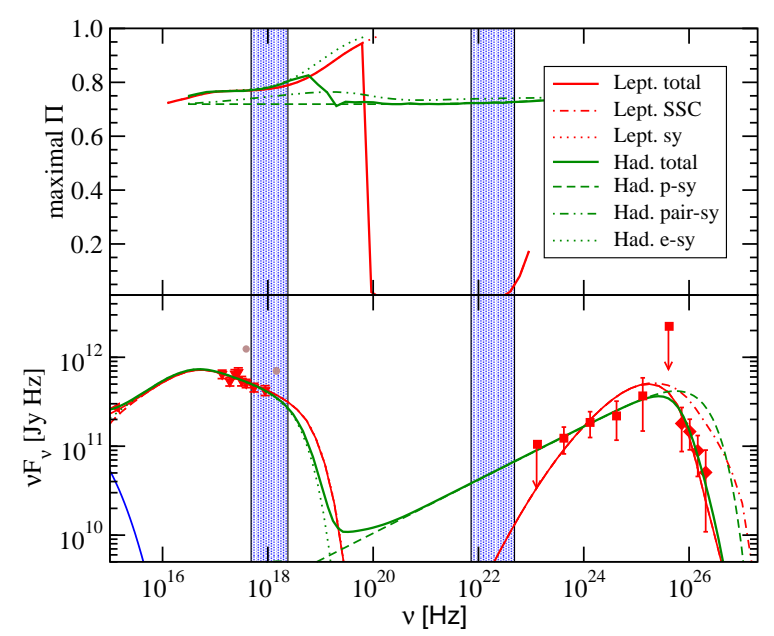

Figure 3. Lower panels: Zoom-in on the UV through $\gamma$-ray SEDs and leptonic (red) and hadronic (green) spectral fits to the IBL 3C66A (left) and the HBL RBS 0413 (right). Top panels: Frequency-dependent maximum degree of polarization corresponding to the spectral fits presented in the lower panels.

actually measured degree of polarization with the theoretical upper limits, will then allow one to re-scale the results from our calculations to arrive at realistic predictions.

In the case of FSRQs and LBLs, it is generally agreed that the near infrared (NIR) - optical emission is dominated by synchrotron radiation. Optical polarimetry will therefore allow one to determine the degree of order/disorder of the magnetic field. X-ray polarimetry may be able to distinguish SSC from proton-synchrotrondominated emission. In the Fermi-LAT regime, the difference between hadronic and leptonic model is expected to be even more obvious, and a positive detection of $\gamma$ ray polarization by Fermi-LAT would strongly favor a hadronic emission scenario. Optical polarimetry of LSP blazars often reveals $\Pi_{\text {sy }}$ (opt) $\sim 10-20 \%$, compared to $\Pi_{\mathrm{sy}}^{\max } \sim 75 \%$ for a typical spectral index of $p \sim 3$. Consequently, a similar degree of X-ray and $\gamma$-ray polarization of $\Pi_{\mathrm{psy}} \sim 10-20 \%$ may be expected for quasars in the case of hadronic emission, which is at the borderline of the capabilities of existing polarimeters.

In the case of IBLs and HBLs, where the X-rays (in IBLs only the soft X-rays) are dominated by synchrotron emission, the degree of order/disorder of the magnetic field may be determined directly from X-ray polarimetry by compmaring the measured $\Pi_{\text {sy }}$ to the theoretical maximum from synchrotron polarization, as evaluated by our calculations. As in the case of FSRQs, the resulting rescaling factor may be used to assess whether the expected $\gamma$-ray polarization is within the capabilities of Fermi-LAT to determine polarization, and if so, hadronic emission is expected to reveal itself through a high degree of polarizaiton.

We should point out here that HBLs typically exhibit very hard photon spectra in the Fermi-LAT range, with very low photon fluxes below $200 \mathrm{MeV}$. The detection of $\gamma$-ray polarization in HBLs therefore seems infeasible in the near future. It has been suggested that HBLs may be prime targets for X-ray polarimeters since they tend to be the brightest blazars in X-rays. However, unfortunately, leptonic and hadronic models agree on the interpretation of the X-ray emission of HBLs as due to electron synchrotron radiation, and therefore make identical predictions for the X-ray polarization. Thus, X-ray polarimetry of HBLs is not expected to aid in distinguishing leptonic from hadronic emission, given current $\gamma$-ray polarimetry capabilities. Such diagnostics would require substantially increased sensitivity and/or $\gamma$-ray polarimetry at energies much above $200 \mathrm{MeV}$.

\section{References}

[1] Aharonian, F. A., New Astron., 5, 377 (2000)

[2] Beilicke, M. G., et al., AIP Conf. Proc., 1505, 805 (2012)

[3] Blażejowski, M., et al., ApJ, 545, 107 (2000)

[4] Bloom, S. D., \& Marscher, A. P., ApJ, 461, 657 (1996)

[5] Böttcher, M., Proc. of "Fermi Meets Jansky", MPIfR, Bonn, Eds. T. Savolainan, E. Ros, R. W. Porcas, \& J. A. Zensus; p. 41 (2012)

[6] Böttcher, M., Harris, D., \& Krawczynski, H. (Eds.) Relativistic Jets from Active Galactic Nuclei (WileyVCH, Berlin, 2012), Chapter 2, p. 19 - 38

[7] Böttcher, M., Reimer, A., Sweeney, K., \& Prakash, A., ApJ, 768, 54 (2013)

[8] Bonometto, S., \& Saggion, A., A\&A, 23, 9 (1973)

[9] Bühler, R., et al., talk at SCINEGHE 2010, Trieste (2010), http://www.rolfbuehler.net/index_talks.html

[10] Dean, A. J., et al., Science, 321, 1183 (2008)

[11] Dermer, C. D., \& Schlickeiser, R., ApJ, 416, 458 (1993)

[12] Dimitrakoudis, S., et al., A\&A, 546, 120 (2012)

[13] Forot, M., et al., ApJL, 688, L29 (2008)

[14] Kelner, S. R., \& Aharonian, F. A., Phys. Rev. D, 78, 034013 
[15] Mannheim, K., \& Biermann, P. L., A\&A, 253, L21 (1992)

[16] Maraschi, L., Celotti, A., \& Ghisellini, G., ApJ, 397, L5 (1992)

[17] Mücke, A., \& Protheroe, R. J., Astropart. Phys., 18, 593 (2001)

[18] Mücke, A., et al., Astropart. Phys., 18, 593 (2003)

[19] Orsi, S., ASTRA, 7, 43 (2011)

[20] Pearce, M., et al., IEEE Nuclear Science Symp. (arXiv:1211.5094)
[21] Reimer, A., Böttcher, M., \& Postnikov, S., ApJ, 630, 186 (2005)

[22] Rybicki, G. B., \& Lightman, A. P., Radiative Processes in Astrophysics (Wiley, New York, 1985)

[23] Schlickeiser, R., A\&AS, 120, 481 (1996)

[24] Sikora, M., Begelman, M., \& Rees, M., ApJ, 421, 153 (1994)

[25] Spanier, F., \& Weidinger, M., IJMPS, 8, 293 (2012)

[26] Zhang, H., \& Böttcher, M., ApJ, in press (2013) 